Denise Loyola Silva

Value Stream Mapping: Uma Revisão Sistemática da Literatura

Dissertação de Mestrado (opção profissional)

Dissertação apresentada como requisito parcial para obtenção do grau de Mestre pelo Programa de PósGraduação em Engenharia de Produção do Departamento de Engenharia Industrial da PUC-Rio.

Orientador: Prof. Fernando Luiz Cyrino Oliveira Co-orientador: Prof. Antônio Márcio Tavares Thomé

Rio de Janeiro 


\section{Value Stream Mapping: Uma Revisão Sistemática da Literatura}

Dissertação apresentada como requisito parcial para obtenção do grau de Mestre (opção profissional) pelo Programa de Pós-Graduação em Engenharia de Produção do Departamento de Engenharia Industrial do Centro Técnico Científico da PUC-Rio. Aprovada pela Comissão Examinadora abaixo assinada.

Prof. Fernando Luiz Cyrino Oliveira Orientador

Departamento de Engenharia Industrial - PUC-Rio

Prof. Antônio Márcio Tavares Thomé Co-orientador Departamento de Engenharia Industrial - PUC-Rio

Prof. José Eugênio Leal Departamento de Engenharia Industrial - PUC-Rio

Prof. Luiz Felipe Roris Rodriguez Scavarda do Carmo Departamento de Engenharia Industrial - PUC-Rio

Prof. José Eugênio Leal Coordenador(a) Setorial do Centro Técnico Científico - PUC-Rio 
Todos os direitos reservados. É proibida a reprodução total ou parcial do trabalho sem autorização do autor e do orientador.

\section{Denise Loyola Silva}

Graduou-se em Engenharia de Produção pela Universidade Veiga de Almeida em 2012. Participou de Projeto de Iniciação Cientifica referente a Metodologia Lean-SixSigma na mesma Instituição e possui publicações acadêmicas em congressos nacionais referente ao tema. Atua em projetos de Lean Manufacturing, Gestão de Estoque e Previsão de Demanda no Mercado de Lubrificantes.

Ficha Catalográfica

Silva, Denise Loyola

Value Stream Mapping: Uma Revisão Sistemática da Literatura / Denise Loyola Silva; orientador: Fernando Luiz Cyrino Oliveira; co-orientador: Antônio Marcio Tavares Thomé. - 2015.

110 f. : il. (color.) ; $30 \mathrm{~cm}$

Dissertação (mestrado) - Pontifícia Universidade Católica do Rio de Janeiro, Departamento de Engenharia Industrial, 2015.

Inclui bibliografia

1. Engenharia industrial - Teses. 2. Revisão sistemática da literatura. 3. Quadro conceitual de referência. 4. Redução de desperdícios. I. Oliveira, Fernando Luiz Cyrino. II. Thomé, Antônio Márcio Tavares. III. Pontifícia Universidade Católica do Rio de Janeiro. Departamento de Engenharia Industrial. IV. Título. 


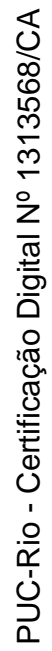

Para minha mãe. 


\section{Agradecimentos}

Agradeço a Deus, por iluminar os meus caminhos e me dar força para seguir em frente com os meus objetivos.

Agradeço, em especial, de todo o meu coração aos meus pais, Donilha Loyola do Amaral † e Carlos Almir do Amaral $\uparrow$, por serem a minha fonte inesgotável de energia, pelo amor incondicional e pela razão da minha constante luta em me tornar uma pessoa melhor a cada dia. Obrigado pelos exemplos de força e coragem que fizeram com que eu nunca desistisse dos meus sonhos.

Ao meu amado esposo, Aluisio, pelo acompanhamento mais que intenso, por todo amparo ao longo processo de construção desta dissertação e por dividir tantos outros sonhos e projetos. Sem o seu amparo, sem sua confiança e sem o seu amor, eu não teria chegado até aqui.

Agradeço ao meu orientador Prof. Fernando Luiz Cyrino Oliveira, pela grande confiança e pelas valiosas contribuições para o presente trabalho. Gostaria de fazer um agradecimento, em especial, ao meu co-orientador Prof. Márcio Thomé pela sua grande dedicação e apoio. Sua experiência e conhecimentos repassados durante todo o desenvolvimento dessa dissertação, foram fundamentais para que este trabalho fosse efetuado com sucesso.

Aos professores presentes na banca, a todos os professores do Departamento de Engenharia Industrial e a PUC-RJ pelos ensinamentos e orientações, contribuindo para a minha evolução e o meu desenvolvimento acadêmico.

Aos meus amigos, em especial, Danielle Motta e Layza Segurasso que, especialmente neste último ano, me confortaram, dando paz e alguma serenidade no momento do falecimento da minha mãe e aos meus amigos do curso de mestrado, em especial, Natália Oliveira, pelos momentos divididos juntos. 
Obrigado por dividirem comigo as angústias e as alegrias ao longo desses dois anos.

Por fim, o meu profundo agradecimento a todos aqueles que contribuíram para a concretização desta dissertação. 


\section{Resumo}

Silva, Denise Loyola; Oliveira, Fernando Luiz Cyrino; Thomé, Antônio Márcio Tavares. Value Stream Mapping: Uma Revisão Sistemática da Literatura. Rio de Janeiro, 2015. 110p. Dissertação de Mestrado (opção profissional) - Departamento de Engenharia Industrial, Pontifícia Universidade Católica do Rio de Janeiro.

O Value Stream Mapping (VSM) é uma das ferramentas da Produção Enxuta que tem sido amplamente discutida na literatura para mapeamento de processos de produção e serviços com foco em redução de desperdícios e de tempo sem valor agregado. Apesar da crescente quantidade de publicações sobre o tema, a literatura acadêmica evidencia a ausência de uma pesquisa sistemática que apresente o estado da arte. Desta forma, esta dissertação, por meio de uma revisão sistemática e criteriosa da literatura, objetiva reunir informações sobre o VSM de forma a identificar suas principais características, tendências e oportunidades para novos desdobramentos e pesquisas futuras. A revisão inclui 263 resumos e 91 artigos selecionados para a revisão e classificação. O estudo propõe uma síntese, em forma de um quadro conceitual de referência, que apresenta como VSM é adotado em determinados contextos e motivações, traçando um melhor entendimento de como a ferramenta é implementada e as respectivas saídas e resultados que podem ser gerados. Percebeu-se que o VSM está se espalhando rapidamente para o setor de serviços, sobretudo de health care e logística. No entanto, estudos relatam que a implementação do VSM, na prática, é um processo demorado. Problemas relacionados à resistência nas melhorias, que envolvem mudanças na força de trabalho e exigência de recursos internos escassos, têm estimulado alguns pesquisadores a questionar o uso da ferramenta. Esta dissertação contribui para uma melhor compreensão do tema e fornece indicações para futuras pesquisas e práticas do VSM.

\section{Palavras-chave}

Revisão sistemática da literatura; quadro conceitual de referência; redução de desperdícios. 


\section{Abstract}

Silva, Denise Loyola; Oliveira, Fernando Luiz Cyrino (Advisor); Thomé, Antônio Márcio Tavares (Co-Advisor). Value Stream Mapping: A Systematic Review of the Literature. Rio de Janeiro, 2015. 110p. MSc. Dissertation - Departamento de Engenharia Industrial, Pontifícia Universidade Católica do Rio de Janeiro.

The Value Stream Mapping (VSM) is one of the Lean Production tools that has been widely discussed in the literature for mapping production processes and services with a focus on reduction of waste and non-value added time. Despite the growing number of publications on the subject, the academic literature reveals the absence of systematic research to present the state of the art. Thus, this dissertation, through a systematic and thorough review of the literature, aims to gather information on the VSM to identify its main characteristics, trends and opportunities for new developments and future research. The review includes 263 abstracts and 91 articles selected for review and classification. The study proposes a synthesis in the form of framework, presenting as VSM is adopted in certain contexts and motivations, tracing a better understanding of how the tool is implemented and their outputs and outcomes that can be generated. It was noticed that the VSM is rapidly spreading to the service sector, especially health care and logistics. However, studies have reported that the implementation of the VSM in practice is a time consuming process. Problems related to resistance in improvements involving changes in the workforce and demand for limited internal resources, have stimulated some researchers to question the use of the tool. This dissertation contributes to a better understanding of the topic and provides directions for future research and VSM practices.

\section{Keywords}

Systematic literature review; reference conceptual framework; reduction of waste. 


\section{Sumário}

1. Introdução 14

$\begin{array}{ll}\text { 1.1. Descrição do trabalho } & 17\end{array}$

$\begin{array}{lr}\text { 2. Metodologia } & 18\end{array}$

3. Análise descritiva 25

3.1. Identificação do estudo 25

3.2. Descrição do VSM 32

3.3. Tipo de estudo 33

3.3.1. Estudo de caso 36

§ $\quad$ 3.3.2. Estudo de investigação - survey 44

$\begin{array}{ll}\text { 3.3.3. Modelo matemático } & 47\end{array}$

3.3.4. Pesquisa-ação $\quad 47$

3.3.5. Revisão da literatura 48

3.3.6. Simulação 48

3.3.7. Multimétodo $\quad 51$

4. Resultado e discussões 53

4.1. Síntese da revisão da literatura - framework 53

4.2. Descrição do estudo 56

4.2.1. Objetivos e metas de negócios 56

4.2.2. Contexto 58

4.2.3. Motivação $\quad 64$

4.2.4. Implementação $\quad 70$

4.2.5. Saídas e resultados 80

5. Conclusões e considerações finais $\quad 84$

$\begin{array}{lr}\text { 6. Referências bibliográficas } & 87\end{array}$ 
7. Apêndices 103

7.1. Apêndice A - Descrição do tipo de estudo 103

7.1.1. Estudo de caso 103

7.1.2. Estudo de investigação - survey 103

7.1.3. Modelo matemático 104

7.1.4. Pesquisa-ação 104

7.1.5. Revisão da literatura 104

7.1.6. Simulação 105

7.1.7. Multimétodo 105

7.2. Apêndice $B-T i p o$ de informações coletadas no artigos selecionados para o estudo do VSM 106

7.3. Apêndice $\mathrm{C}$ - Resultados para primeira avaliação da revisão da literatura sobre Value Stream Map (VSM)* 107

ㄱ. 7.4. Apêndice D - Resultados para segunda avaliação da revisão da

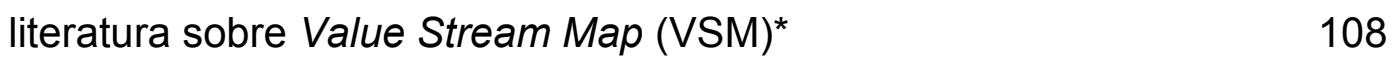
7.5. Apêndice $E$ - Resultados para terceira avaliação da revisão da literatura sobre Value Stream Map (VSM)* 109 7.6. Apêndice $F-$ Resultados para quarta avaliação da revisão da literatura sobre Value Stream Map (VSM)* 


\section{Lista de figuras}

Figura 1 - Resultado da busca por cinco bases de dados

Figura 2 - Descrição do resultado da pesquisa bem como o número de artigos selecionados por revisão

Figura 3 - Comparação entre o número de artigos obtidos na busca e $\mathrm{n}^{\circ}$ de artigos selecionados

Figura 4 - Número de documentos recuperados para a análise de revisão sistemática

Figura 5 - Percentual da abordagem metodológica empregada nas publicações sobre VSM

Figura 6 - Sínteses da revisão da literatura do VSM

Figura 7 - Percentual de publicações por região geográfica

Figura 8 - Percentual de publicações por ano e região geográfica 59

Figura 9 - Percentual de publicações por tipo de setor 62

Figura 10 - Percentual de publicações por ano e tipo de setor 63

Figura 11 - Percentual de publicações por tipo de produção 64

Figura 12 - Percentual da abordagem do VSM nas publicações 


\section{Lista de tabelas}

Tabela 1 - Descrição das bases de dados 19

Tabela 2 - Resumo de resultados do coeficiente de concordância 23

Tabela 3 - Resumo de resultados dos artigos 25

Tabela 4 - Descrição das publicações selecionadas por metodologia utilizada 35

Tabela 5 - Revisão dos artigos de estudo de caso 37

Tabela 6 - Revisão dos artigos de estudo de investigação-survey $\quad 45$

Tabela 7 - Revisão dos artigos de modelo matemático 47

Tabela 8 - Revisão dos artigos de simulação 49

Tabela 9 - Revisão dos artigos de multimétodo 51

Tabela 10 - Descrição do tipo de indústria e sua respectiva

Tabela 11 - Descrição das categorias de motivação de acordo com os autores

Tabela 12 - Descrição dos autores de acordo com a ferramenta de apoio

Tabela 13 - Descrição do resultado da pesquisa por autor

Tabela 14 - Descrição das informações coletados nos artigos selecionados para o estudo do VSM 
" Quem sabe concentrar-se numa coisa e insistir nela como único objetivo, obtém, ao fim e ao cabo, a capacidade de fazer qualquer coisa."

Mahatma Gandhi 